\title{
La voz de los estudiantes de la titulación de Educación Primaria sobre el Trabajo Fin de Grado
}

\author{
Javier Rodríguez Moreno ${ }^{1}$ \\ ORCID: 0000-0002-5890-3654 \\ $M^{2}$ Dolores Molina Jaén ${ }^{1}$ \\ ORCID: 0000-0001-6010-6382 \\ $\mathrm{M}^{\mathrm{a}}$ Jesús Colmenero Ruiz ${ }^{1}$ \\ ORCID: 0000-0003-3559-2512
}

\section{Resumen}

El marco normativo español tras la entrada en vigor del Espacio Europeo de Educación Superior, hace necesaria una revisión del planteamiento actual que se está otorgando a la asignatura Trabajo Fin de Grado -TFG-. El objetivo de este trabajo ha sido analizar la voz del alumnado que ha finalizado el Trabajo Fin de Grado en la titulación de Educación Primaria en base a su proceso de enseñanza-aprendizaje, tutorización, evaluación y aplicabilidad del mismo y que ha cursado dicha asignatura en la Universidad de Jaén y en el Centro Universitario SAFA de Úbeda (España). Para ello, se ha analizado el tratamiento que hacen del TFG tanto la comunidad científica como la normativa vigente al respecto. La muestra participante ha sido de 237 alumnos y para tal efecto se ha diseñado un cuestionario ad hoc como instrumento de recogida de información, con una escala tipo Likert compuesta por cuatro dimensiones y 33 items en total. Los resultados muestran que el TFG no cumple las expectativas del alumnado en las dimensiones analizadas de tutorización, aplicabilidad, evaluación y desarrollo del proceso enseñanza- aprendizaje, alcanzando estas medias que no sobrepasan, en el mejor de los casos, el 30\% de valoración. Las conclusiones ponen de manifiesto que queda todavía camino por recorrer para que TFG y calidad sean sinónimos.

\section{Palabras clave}

Trabajo fin de grado - Educación primaria - Alumnado - Opinión.

1- Universidad de Jaén, Jaén, España. Contactos: jrmoreno@ujaen.es; lomolin@hotmail.com; mjruiz@ujaen.es. 


\section{The voice of students in the degree of Primary Education about the final degree project}

\section{Abstract}

The Spanish legal framework after the approval of regulation of the European Higher Education Area requires a revision of the current approach being granted to the subject Final Degree Project - TFG. The objective of this work has been to analyse the voice of students who have completed the Final Degree Project in the degree of Primary Education based on their teaching-learning process, tutoring, evaluation and applicability thereof and those who completed this subject in the University of Jaén and the University Centre SAFA of Úbeda (Spain). To do so, we analysed the treatment of the TFG by both the scientific community and the relevant current regulations. Sample consisted of 237 students so an ad hoc questionnaire was designed as a tool to collect information, using a Likert scale composed of four dimensions and 33 items in total. The results show that the TFG does not meet the expectations of the students in the analysed dimensions of tutoring, applicability, evaluation and development of the teaching-learning process, as they reach average values not higher, among the best cases, 30\% of assessment. The conclusions show that there is still a long way to go before TFG and quality will be synonyms.

\section{Keywords}

Final degree project - Primary education - Students - Opinion.

\section{Introducción}

La convergencia de sistemas educativos universitarios supuso el comienzo del Espacio Europeo de Educación Superior -EEES-, desencadenando una serie de reformas a nivel curricular, organizativo y estructural (DÍEZ et al., 2011). En nuestro país, los estudios de grado y máster se rigen por el Real Decreto 1393/2007 (ESPAÑA, 2007), por el que se establece la ordenación de las enseñanzas universitarias oficiales y por el Real Decreto 43/2015 (ESPAÑA, 2015), que lo modifica. En dicha normativa, se regula que dichos estudios deben ser finalizados con el diseño, elaboración y defensa de un Trabajo de Fin de Grado (TFG) o Trabajo Fin de Máster (TFM), en el que el alumnado debe demostrar la adquisición de las competencias exigidas.

Por su parte, la Orden ECI/3857/2007 (ESPAÑA, 2007) estructura el Grado en Educación Primaria en tres módulos, el primero de formación básica, donde el alumnado desarrolla contenidos de carácter sociológicos, psicológicos y pedagógicos. En el segundo módulo, didáctico y disciplinar, se trabajan contenidos propios de las áreas disciplinares y su didáctica. Por último, en el Practicum, donde se especifican las competencias que se deben adquirir en el periodo de prácticas. Así mismo, en este tercer módulo, es donde se encuadra el Trabajo Fin de Grado -TFG- con la adquisición de los aprendizajes propios de 
cada titulación y, donde el alumnado debe demostrar las competencias relativas a los tres módulos citados anteriormente. A partir de este marco jurídico, la Universidad de Jaén estableció su normativa propia sobre el Trabajo Fin de Grado -aprobada en 2012- y, más concretamente, la Facultad de Humanidades y Ciencias de la Educación aprobó, el año 2017, un nuevo reglamento adaptado a los Trabajos Fin de Grado propios de nuestra Facultad.

Si bien es cierto que se trata de un tema relativamente nuevo, existen trabajos muy interesantes que nos han servido de guía acerca de cómo se está actuando a nivel general en esta temática, nos gustaría destacar los de Bartolomé y otros autores (2012), Bonilla y Martín (2012); Fondevila y Olmo (2013); García y Martínez (2012), Gutiérrez y López (2012), Mateo (2009), Muñiz y Fonseca (2009), Pérez y Martínez (2017), Reyes y Díaz (2017), Sánchez y otros autores (2015), Villa y Poblete (2007), existe, además, gran variedad de publicaciones sobre aspectos más específicos y diversos del TFG como las de Ferrer, Carmona y Soria (2012), González, León, Peñalba (2014), Latorre (2011), Muñoz (2015), Rodríguez (2012), Rullán y otros autores (2010), Vera y Briones (2015). Más concretamente, y centrándonos en la finalidad principal de nuestra investigación, queremos destacar los trabajos de De Vaus (2002), Lavrakes (2008), Moreno y Hernández (2015), De Pro, Sánchez y Valcárcel (2013), Tur y otros autores (2013), Valderrama y otros autores (2010), Pepper, Webster y Jenkins (2001) y Zairi (2000).

Por tanto, una asignatura que ha nacido con la peculiaridad de ser un trabajo en sus diferentes versiones debe posibilitar al alumnado una formación de calidad en sus respectivas titulaciones. Hasta el momento la experiencia y la investigación nos suscitan algunas dudas (AMADOR; SERRANO, 2017) y es por ello que se plantea este trabajo: debemos mejorar bastante en el planteamiento de esta asignatura para que verdaderamente sea un trabajo que aumente la calidad de la educación.

De las investigaciones que aportan soluciones, destacamos el trabajo de Rodríguez y otros autores (2017), que plantea una tutoría piramidal, donde sería positivo desarrollar una sesión en gran grupo, en grupos pequeños y luego de forma individual, idea que ya planteaba Zabalza (2006). En definitiva, se trata de crear un ambiente de intercambio de experiencias, dificultades, miedos, posibilidades, que origine un clima de trabajo donde los estudiantes por si solos sean capaces de provocar aprendizaje en relación al TFG. Otras investigaciones, siendo conscientes de las dificultades de la metodología, proponen estrategias y la búsqueda de soluciones para mejorar esta asignatura (SÁNCHEZ et al., 2015). Por último, de una manera o de otra, el trabajo del TFG debe convertirse en una oportunidad y no en un trámite (SOTOS, 2015) y para ello propone trabajo en equipo, formación investigadora (el gran déficit) y generar saber pedagógico que le sirva para la práctica.

\section{Método}

\section{Objetivos}

El objetivo principal que pretende esta investigación ha sido analizar la voz del alumnado que ha finalizado la asignatura Trabajo Fin de Grado sobre su proceso de enseñanza-aprendizaje, tutorización, evaluación y aplicabilidad del mismo. Para ello, nos vamos a centrar en los tres elementos clave de cualquier proceso investigador como son 
el instrumento de recogida de datos, los participantes y el contexto de aplicación y, por último, el procedimiento utilizado.

En la investigación se plantearon los siguientes objetivos específicos:

- Comparar si existen diferencias según el género de los estudiantes.

- Analizar las desigualdades en las respuestas según la edad de los estudiantes.

- Conocer según la nota de acceso a la universidad, las aportaciones en las distintas dimensiones.

- Identificar si existen diferencias entre los estudiantes según el tipo de TFG.

- Comprobar la variedad en la voz de los estudiantes según la nota media del grado de los mismos.

- Establecer las diversidades que se pueden dar según el tipo de centro de realización del grado de los participantes.

\section{Población y muestra}

La población objeto de estudio está compuesta por 361 estudiantes del Grado en Educación Primaria, matriculados el $83.1 \%$ en la Universidad de Jaén y el 16.9\% en la Escuela privada de Magisterio Sagrada Familia de Úbeda. Para la selección de sujetos se utilizó un tipo de muestreo no probabilístico de tipo accidental o casual (LATORRE; DEL RINCÓN; ARNAL, 2003); finalmente, se han adscrito a la muestra todos aquellos sujetos que accedieron a responder el cuestionario $(n=237)$. Para el cálculo de la muestra participante se utilizó la fórmula para poblaciones con menos de 100.000 sujetos con un nivel de confianza del 99\% y un error de estimación máximo de un 4\%.

De los 237 estudiantes el 65.8\% son mujeres y el resto (34.2\%) son hombres. En cuanto a la edad media de los encuestados es 24 años, siendo el rango de edad de los encuestados desde los 21 años a los 41 años y siendo el valor que más se repite en los encuestados 22 años.

\section{Instrumento}

En el presente trabajo se ha utilizado una metodología cuantitativa descriptiva a través de la técnica de la encuesta. Para ello, se diseñó un cuestionario ad hoc como instrumento de recogida de información. Se trataba de una escala tipo Likert de 4 puntos donde las opciones de respuesta oscilaron entre 1 y 4 (siendo 1, nada; 2, poco; 3, bastante; y 4, totalmente). El cuestionario estaba estructurado en dos grandes bloques; por un lado, se pretendía caracterizar los participantes en esta investigación (género, edad, universidad de matriculación, nota de acceso a la universidad, tipo de TFG realizado y nota media en el grado) y un segundo, constituido por 33 ítems acerca de las opiniones que tiene el alumnado sobre el Trabajo Fin de Grado y quedó configurado en cuatro dimensiones: Proceso de enseñanza-aprendizaje del TFG (4 ítems), Tutorización (11 ítems), Evaluación (9 ítems) y Aplicabilidad del TFG (9 ítems). 
En cuanto a la validez del contenido, se realizó mediante un juicio de expertos y la aplicación de una prueba piloto. Así, se solicitó a siete docentes de distintas universidades españolas del área de Didáctica y Organización Escolar que llevasen a cabo una valoración del instrumento e indicaran el grado de adecuación, pertenencia y precisión del mismo al objeto de estudio. En líneas generales, las evaluaciones de los expertos mostraron que la mayoría de los ítems eran adecuados para esta investigación, tan solo tuvimos que suprimir cinco de ellos y modificar aspectos gramaticales en otros dos.

Una vez realizado el juicio de expertos, se procedió a aplicar una prueba piloto a veinte estudiantes del Grado de Primaria de la Universidad de Jaén y de la Escuela de Magisterio Sagrada Familia de Úbeda. Así, los datos de adecuación muestral KaiserMeyer-Olkin (.815) y la prueba de esfericidad de Barlett $(\chi 2=1724,24$; gl=528; $p<.05$ $(\mathrm{p}=0.000)$ indican que se cumplen las condiciones para la realización del análisis factorial. Aplicando el método de extracción de componentes principales y el método de rotación de Varimax con normalización Kaiser, los resultados del análisis apuntan la existencia de cuatro factores que explican el 71.94\% de la varianza total.

Así mismo, se contrastó la variabilidad (mediante la varianza $\sigma^{2}$ ) entre los ítems y la fiabilidad del instrumento analizado por medio del coeficiente Alpha de Cronbach que es excelente, al ser el coeficiente .927 , lo que indica una adecuada consistencia interna de la escala.

González, Abad y Levy (2006) afirman que los estadísticos de contraste z(G1, p) y z(G2, p) obtenidos a partir de G1, p y G2, p se distribuyen asintóticamente según una normal estándar por los que su interpretación es semejante a la ya comentada para los estadísticos de asimetría y curtosis univariante $\mathrm{z}(\mathrm{G} 1)$ y $\mathrm{z}(\mathrm{G} 2)$ : valores experimentales que en valor absoluto sean mayores que 1.96 permiten rechazar a un nivel de significación del 5\% las respectivas hipótesis nulas de distribución multivariante simétrica y mesocúrtica. Así mismo, también se puede realizar un contraste conjunto de simetría y mesocurtosis multivariantes utilizando el estadístico $\mathrm{kp} 2=[\mathrm{z}(\mathrm{G} 1, \mathrm{p})] 2+[\mathrm{z}(\mathrm{G} 2, \mathrm{p})] 2$, que se aproxima a una distribución $\chi^{2}$ con dos grados de libertad y que también se interpreta de forma análoga al estadístico conjunto k2 de normalidad univariante, es decir, se rechaza la hipótesis nula para valores experimentales mayores que 5.99 dado un nivel de significación del 5\%.

Tabla 1 - Coeficientes de asimetría y curtosis de las dimensiones

\begin{tabular}{|c|c|c|c|c|c|}
\hline \multicolumn{2}{|c|}{ Coeficientes } & Proceso de E-A del TFG & Tutorización & Evaluación & Aplicabilidad TFG \\
\hline \multirow{2}{*}{ Asimetría } & Estadístico & -.49 & -.71 & -.46 & -.41 \\
\hline & Error típ. & .27 & .27 & .53 & .27 \\
\hline \multirow{2}{*}{ Curtosis } & Estadístico & -.25 & -.41 & .01 & -.34 \\
\hline & Error típ. & .53 & .27 & .53 & .53 \\
\hline
\end{tabular}

Fuente: Elaboración propia.

Si se divide cada uno de los coeficientes entre su respectivo error típico hemos calculado los estadísticos z(G1) y z(G2), y sumando los cuadrados de estos últimos 
hallamos el valor experimental del estadístico de contraste conjunto k2 (GONZÁLEZ;ABAD; LEVY, 2006), siendo los resultados obtenidos los siguientes (tabla 2):

Tabla 2 - Cálculo del coeficiente conjunto de asimetría y curtosis de las dimensiones

\begin{tabular}{c|cc:c:c:cc}
\hline Contraste & Proceso de E-A del TFG & Tutorización & Evaluación & Aplicabilidad TFG \\
\hline Asimetría: $\mathrm{z}(\mathrm{G} 1)$ & -1.82 & -2.63 & -.86 & -1.50 \\
\hline Curtosis: $\mathrm{z}(\mathrm{G} 2)$ & -.47 & -1.52 & .02 & -.63 \\
\hline Conjunto: $\mathrm{k} 2$ & 3.52 & 9.19 & & .74 & 2.66 \\
\hline
\end{tabular}

Fuente: Elaboración propia.

Como se puede observar, el contraste conjunto de asimetría y curtosis, indica que sólo pueden considerarse como normales las dimensiones Proceso de enseñanzaaprendizaje del TFG, Evaluación y aplicabilidad del TFG.

\section{Procedimiento de recogida y análisis de datos}

Para la cumplimentación del cuestionario por parte de los participantes, se les envió al alumnado matriculado en la asignatura Trabajo Fin de Grado -TFG- el enlace al instrumento que tenían que rellenar on-line al finalizar la asignatura en el curso académico 2016-17 y las aclaraciones procedimentales, asegurando en todo momento la confidencialidad y anonimato de los datos recogidos.

Para el análisis de datos, se utilizó el paquete estadístico SPSS.24, realizándose un análisis descriptivo de las variables independientes, en concreto, de los estadísticos básicos moda, mediana, media, desviación típica, error típico de la media, así como el valor mínimo y máximo de las puntuaciones obtenidas por los participantes.

Asumiendo la normalidad de las distribuciones (MARTÍNEZ et al., 2014) e independencia, se aplicó la prueba T de Student para muestras independientes. Con ello, se analizó si existen diferencias significativas entre las respuestas del alumnado en función del género, edad, nota de acceso a la universidad, tipo de TFG, nota media del grado y tipo de centro. Completando la información para conocer el tamaño del efecto de las diferencias encontradas, se calculó el estadístico $d$ de Cohen (1988). Además, se ha establecido las relaciones tanto lineal como monótona entre las distintas dimensiones del cuestionario y distintos diagramas de dispersión para representar las distintas combinaciones entre las dimensiones.

\section{Resultados}

Entre los posibles valores que el alumnado puede puntuar en el total del cuestionario (33 ítems), podemos observar que la media de la dimensión Proceso de enseñanza-aprendizaje se encuentra en 11.30 (d.t.=3.14), siendo 12 el valor más elegido (gráfico 1). En cuanto a la media de la dimensión Tutorización se encuentra en 32.10 (d.t=7.17) (gráfico 2). La dimensión Evaluación tiene como media 26.45 
(d.t=4.54) (gráfico 3). Y, la dimensión Aplicabilidad del TFG tiene como valor medio 25.26 (d.t=6.97) (gráfico 4).

Tabla 3 - Descriptivos correspondientes a las dimensiones del cuestionario

\begin{tabular}{|c|c|c|c|c|c|c|c|}
\hline Factor & $\mathrm{N}$ & Mo & $\mathrm{Me}$ & $\bar{x}$ & d.t & E.T.M & Mín - Máx \\
\hline Proceso de E-A del TFG & 237 & 12 & 12 & 11.30 & 3.14 & .35 & $4-16$ \\
\hline Tutorización & 237 & 39 & 33 & 32.10 & 7.17 & .81 & $12-42$ \\
\hline Evaluación & 237 & 25 & 27 & 26.45 & 4.54 & .51 & $13-35$ \\
\hline Aplicabilidad del TFG & 237 & 20 & 25 & 25.26 & 6.97 & .78 & $9-36$ \\
\hline
\end{tabular}

Fuente: Elaboración propia.

Gráfico 1 - Proceso de E-A TFG

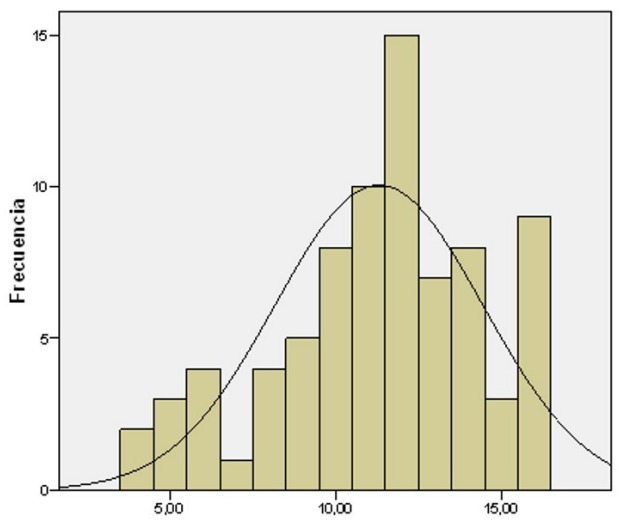

Gráfico 3 - Evaluación

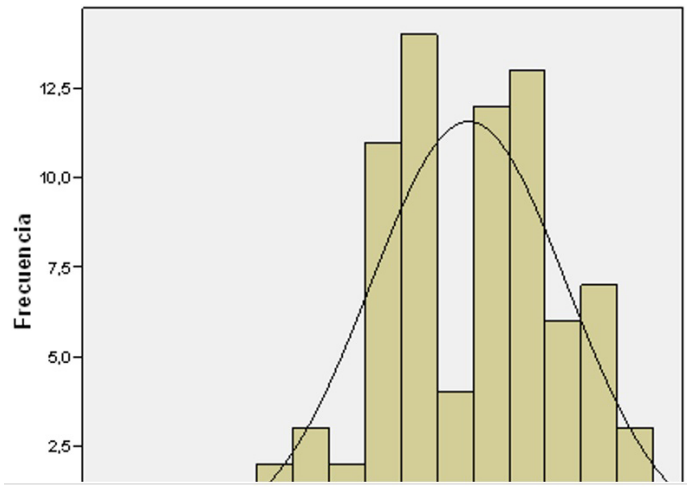

Gráfico 2 - Tutorización

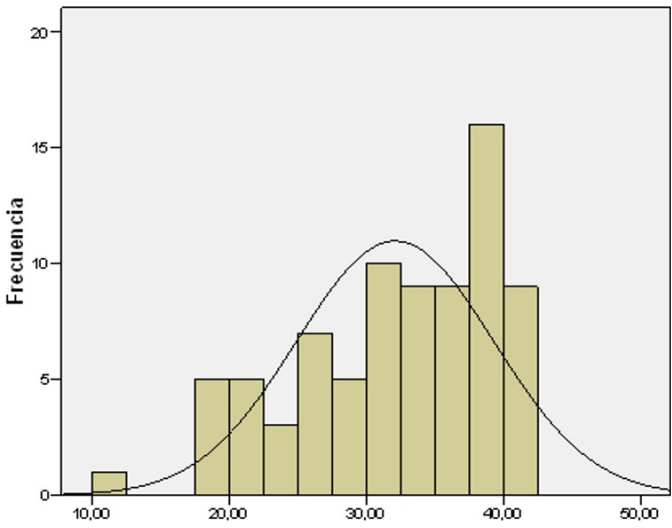

Gráfico 4 - Aplicabilidad TFG

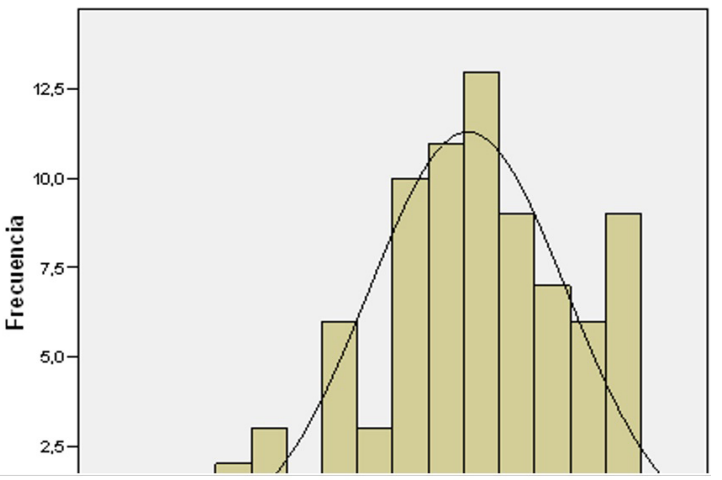

Fuente: Elaboración propia.

\section{Diferencias de valoración del cuestionario según el género}

Las alumnas valoran mejor la dimensión Proceso de enseñanza y de aprendizaje $(=11.35$; d.t.=3.08) que los alumnos $(=11.22$; d.t.=3.30) aunque no existe diferencia 
significativamente ( $\mathrm{p}>.05$ ), también en Tutorización (alumnas =32.77; d.t.=7.13 y alumnos $=30.81$; d.t.=7.22). De la misma manera, las alumnas presentan mayor puntuación $(=27.06$; d.t.=4.24) que los alumnos $(=25.30 ;$ d.t.=4.95) en la dimensión Evaluación, aunque no presentan diferencias estadísticamente significativas $(p=.26>.05)$, (tabla 4$)$.

Tabla 4 - Diferencias en el alumnado según género

\begin{tabular}{|c|c|c|c|c|c|c|c|c|c|c|}
\hline Dimensiones & Genero & $\mathrm{N}$ & $\bar{x}$ & d.t. & E.T.M & $t$ & $\mathrm{gl}$ & $\mathrm{p}$ & $\overline{\mathrm{X}} 1-\overline{\mathrm{X}} 2$ & E.T.D \\
\hline \multirow{2}{*}{$\begin{array}{l}\text { Proceso de } \\
\text { E-A del TFG }\end{array}$} & Hombre & 81 & 11.22 & 3.30 & .63 & \multirow{2}{*}{-.17} & \multirow{2}{*}{77} & \multirow{2}{*}{.87} & \multirow{2}{*}{-.12} & \multirow{2}{*}{.75} \\
\hline & Mujer & 156 & 11.35 & 3.08 & .43 & & & & & \\
\hline \multirow{2}{*}{ Tutorización } & Hombre & 81 & 30.81 & 7.22 & 1.39 & \multirow{2}{*}{-1.15} & \multirow{2}{*}{77} & \multirow{2}{*}{.25} & \multirow{2}{*}{-1.95} & \multirow{2}{*}{1.70} \\
\hline & Mujer & 156 & 32.77 & 7.13 & .99 & & & & & \\
\hline \multirow{2}{*}{ Evaluación } & Hombre & 81 & 25.30 & 4.95 & .95 & \multirow{2}{*}{-1.65} & \multirow{2}{*}{77} & \multirow{2}{*}{.26} & \multirow{2}{*}{-1.76} & \multirow{2}{*}{1.07} \\
\hline & Mujer & 156 & 27.06 & 4.24 & .59 & & & & & \\
\hline \multirow{2}{*}{ Aplicabilidad TFG } & Hombre & 81 & 25.37 & 6.49 & 1.25 & \multirow{2}{*}{.10} & \multirow{2}{*}{77} & \multirow{2}{*}{.10} & \multirow{2}{*}{.16} & \multirow{2}{*}{1.66} \\
\hline & Mujer & 156 & 25.21 & 7.26 & 1.01 & & & & & \\
\hline
\end{tabular}

Gen. = Género; d.t. = Desviación típica; ETM = Error típico de la media; $\mathrm{t}=$ Estadístico $\mathrm{t}$ de Student para muestras independientes; $\mathrm{gl}=\mathrm{Grados}$ de libertad; $p=$ Nivel de significación; X1-X2 = Diferencia de medias; DEE = Diferencias del error estándar; (1) Se asumen varianzas iguales.

Fuente: Elaboración propia.

\section{Diferencias de valoración de la encuesta según la edad}

El alumnado de menos de 30 años valora mejor la dimensión Proceso de enseñanza y de aprendizaje $(=11.39$; d.t. $=3.08)$ que los de 30 y más años $(=10.70$; d.t.=3.59) aunque no existe diferencia significativa $(\mathrm{p}>.05)$. Las encuestados de 30 o más años presentan mayor puntuación $(=34.80$; d.t. $=7.38)$ que los de menos de 30 años $(=31.71$; d.t.=7.11) en Tutorización, aunque no existe diferencia significativa ( $>$.05). De la misma manera, los encuestados de 30 o más años valoran más $(=28.60$; d.t.=3.03) que los de menos de 30 años (=26.14; d.t.=4.65) la dimensión Evaluación, aunque no presentan diferencias estadísticamente significativas $(\mathrm{p}=.11>.05)$. En cambio, los encuestados de menos de 30 años $(=25.87$; d.t $=6.48)$ presentan mayor puntuación que los de 30 o más años $(=21.10$; d.t=9.02) en la dimensión Aplicabilidad TFG, y además existen diferencias significativas con respecto a esta dimensión según la edad, ya que el p-valor es .04 (<.05). Por tanto, como existen diferencias significativas entre la dimensión Aplicabilidad TFG con la edad calculamos el tamaño del efecto (d de Cohen), siendo .61, considerándose un tamaño de efecto moderado (tabla 5). 
Tabla 5 - Diferencias en el alumnado según edad

\begin{tabular}{|c|c|c|c|c|c|c|c|c|c|c|}
\hline Dimensiones & Edad & N & $\bar{x}$ & d.t. & E.T.M & $t$ & $\mathrm{gl}$ & $\mathrm{p}$ & $\bar{x} \bar{x} 1-\bar{X} 2$ & E.T.D \\
\hline \multirow{2}{*}{$\begin{array}{c}\text { Proceso de E-A del } \\
\text { TFG }\end{array}$} & Menor de 30 años & 207 & 11.39 & 3.08 & .37 & \multirow{2}{*}{.65} & \multirow{2}{*}{77} & \multirow{2}{*}{.52} & \multirow{2}{*}{.69} & \multirow{2}{*}{1.06} \\
\hline & De 30 o más años & 30 & 10.70 & 3.59 & 1.14 & & & & & \\
\hline \multirow{2}{*}{ Tutorización } & Menor de 30 años & 207 & 31.71 & 7.11 & .86 & \multirow{2}{*}{-1.28} & \multirow{2}{*}{77} & \multirow{2}{*}{.21} & \multirow{2}{*}{-3.09} & \multirow{2}{*}{2.42} \\
\hline & De 30 o más años & 30 & 34.80 & 7.38 & 2.33 & & & & & \\
\hline \multirow{2}{*}{ Evaluación } & Menor de 30 años & 207 & 26.14 & 4.65 & .56 & \multirow{2}{*}{-1.61} & \multirow{2}{*}{77} & \multirow{2}{*}{.11} & \multirow{2}{*}{-2.46} & \multirow{2}{*}{1.52} \\
\hline & De 30 o más años & 30 & 28.60 & 3.03 & .96 & & & & & \\
\hline \multirow{2}{*}{$\begin{array}{l}\text { Aplicabilidad } \\
\text { TFG }\end{array}$} & Menor de 30 años & 207 & 25.87 & 6.48 & .78 & \multirow{2}{*}{2.07} & \multirow{2}{*}{77} & \multirow{2}{*}{.04} & \multirow{2}{*}{4.77} & \multirow{2}{*}{2.31} \\
\hline & De 30 o más años & 30 & 21.10 & 9.02 & 2.85 & & & & & \\
\hline
\end{tabular}

Edad. = Grupos de edad; d.t. = Desviación típica; ETM = Error típico de la media; $\mathrm{t}=$ Estadístico $\mathrm{t}$ de Student para muestras independientes; $\mathrm{gl}=$ Grados de libertad; $p=$ Nivel de significación; X1-X2 = Diferencia de medias; DEE = Diferencias del error estándar; (1) Se asumen varianzas iguales. Fuente: Elaboración propia.

\section{Diferencias de valoración del alumnado según su nota de acceso a la universidad}

Los estudiantes que acceden a la universidad con una nota inferior al notable2 valoran mejor la dimensión Proceso de enseñanza y de aprendizaje $(=11.45$; d.t.=3.19) que los que acceden con un notable o más $(=11.17$; d.t.=3.11) aunque no existe diferencia significativamente ( $\mathrm{p}>$.05). Los encuestados con una nota de acceso inferior al notable valoran más positivamente la Tutorización $(=32.68 ;$ d.t. $=7.09)$ que los que acceden con un notable o más $(=31.56$; d.t. $=7.30)$, aunque no existe diferencia significativamente ( $\mathrm{p}>.05)$. Así los encuestados que acceden con menos de un notable a la universidad presentan mayor puntuación $(=26.74$; d.t. $=4.35)$ que los que acceden con un notable o superior $(=26.20$; d.t. $=4.74)$ en la dimensión Evaluación, pero no presentan diferencias estadísticamente significativas $(p=.60>.05)$. Por último, los encuestados con nota inferior a notable $(=25.29$; d.t= 7.26) presentan mayor puntuación que los que han accedido con un notable o superior $(=25.24$; d.t=6.77) en la dimensión Aplicabilidad TFG, aunque no presentan diferencias significativas con respecto a esta dimensión, ya que el p-valor es .98 >.05 (tabla 6).

\footnotetext{
2- En España, la nota numérica correspondiente al concepto Notable es 7-8.
} 
Tabla 6 - Diferencias en el alumnado según la nota de acceso a la universidad

\begin{tabular}{|c|c|c|c|c|c|c|c|c|c|c|}
\hline Dimensiones & Nota de acceso & $\mathrm{N}$ & $\bar{x}$ & d.t. & E.T.M & $\mathrm{t}$ & gl & $\mathrm{p}$ & $\bar{X} 1-\bar{X} 2$ & E.T.D \\
\hline \multirow{2}{*}{ Proceso de E-A del TFG } & Menos del notable & 114 & 11.45 & 3.19 & .52 & \multirow{2}{*}{.39} & \multirow{2}{*}{77} & \multirow{2}{*}{.70} & \multirow{2}{*}{.28} & \multirow{2}{*}{.71} \\
\hline & Notable o más & 123 & 11.17 & 3.11 & .49 & & & & & \\
\hline \multirow{2}{*}{ Tutorización } & Menos del notable & 114 & 32.68 & 7.09 & 1.15 & \multirow{2}{*}{-1.28} & \multirow{2}{*}{77} & \multirow{2}{*}{.49} & \multirow{2}{*}{1.12} & \multirow{2}{*}{1.62} \\
\hline & Notable o más & 123 & 31.56 & 7.30 & 1.14 & & & & & \\
\hline \multirow{2}{*}{ Evaluación } & Menos del notable & 114 & 26.74 & 4.35 & .71 & \multirow{2}{*}{-1.61} & \multirow{2}{*}{77} & \multirow{2}{*}{.60} & \multirow{2}{*}{.54} & \multirow{2}{*}{1.03} \\
\hline & Notable o más & 123 & 26.20 & 4.74 & .74 & & & & & \\
\hline \multirow{2}{*}{ Aplicabilidad TFG } & Menos del notable & 114 & 25.29 & 7.26 & 1.18 & \multirow{2}{*}{2.07} & \multirow{2}{*}{77} & \multirow{2}{*}{.98} & \multirow{2}{*}{.05} & \multirow{2}{*}{1.58} \\
\hline & Notable o más & 123 & 25.24 & 6.77 & 1.06 & & & & & \\
\hline
\end{tabular}

Nota $=$ Grupos de nota de acceso a la universidad; d.t. = Desviación típica; ETM = Error típico de la media; $\mathrm{t}$ = Estadístico $\mathrm{t}$ de Student para muestras independientes; $\mathrm{gl}=$ Grados de libertad; $p=$ Nivel de significación; X1-X2 = Diferencia de medias; DEE = Diferencias del error estándar;

(1) Se asumen varianzas iguales.

Fuente: Elaboración propia.

\section{Diferencias de valoración del alumnado según el Tipo de TFG realizado}

Se observa que los encuestados cuyo tipo de TFG es sobre Plan/Proyecto de intervención, innovación e investigación presentan mayor puntuación en la dimensión Proceso de enseñanza y de aprendizaje $(=11.60$; d.t. $=3.00)$ que los que son de Unidad y programación didáctica $(=10.24$; d.t. $=3.47)$ aunque no existe diferencia significativamente $(p=.11>.05)$. Los encuestados cuyo TFG es sobre Plan/ Proyecto de intervención, innovación e investigación presentan mayor puntuación $(=32.61$; d.t.=6.76) que los de Unidad y programación didáctica $(=30.24$; d.t.=8.48) en Tutorización, aunque no existe diferencia significativa $(\mathrm{p}=.23>.05)$. De la misma manera, los encuestados cuyo TFG es de Plan/Proyecto de intervención, innovación e investigación $(=26.65$; d.t.=4.16) que los de Unidad y programación didáctica $(=25.76$; d.t. $=5.82)$ en la dimensión Evaluación, aunque no presentan diferencias estadísticamente significativas $(\mathrm{p}=.48>.05)$. Por último, los encuestados cuyo tipo de TFG trata sobre Plan/Proyecto de intervención, innovación e investigación (=25.95; d.t=6.92) presentan mayor puntuación que los de Unidad y programación didáctica $(=25.95$; d.t=6.92) en la dimensión Aplicabilidad TFG, aunque no presentan diferencias significativas con respecto a esta dimensión, ya que el p-valor es .09 $>.05$ (tabla 7). 
Tabla 7 - Diferencias de valoración según el tipo de TFG

\begin{tabular}{|c|c|c|c|c|c|c|c|c|c|c|}
\hline Dimensiones & Tipo TFG & N & $\bar{x}$ & d.t. & E.T.M & $\mathrm{t}$ & gl & $\mathrm{p}$ & $\overline{\mathrm{X}} 1-\overline{\mathrm{X}} 2$ & E.T.D \\
\hline \multirow{2}{*}{$\begin{array}{c}\text { Proceso de E-A del } \\
\text { TFG }\end{array}$} & Unidad y programación didáctica & 51 & 10.24 & 3.47 & .84 & \multirow[b]{2}{*}{-1.60} & \multirow[b]{2}{*}{77} & \multirow[b]{2}{*}{.11} & \multirow[b]{2}{*}{-1.36} & \multirow[b]{2}{*}{.85} \\
\hline & $\begin{array}{l}\text { Plan/Proyecto de intervención, } \\
\text { innovación e investigación }\end{array}$ & 186 & 11.60 & 3.00 & .38 & & & & & \\
\hline \multirow[b]{2}{*}{ Tutorización } & Unidad y programación didáctica & 51 & 30.24 & 8.48 & 2.06 & \multirow[b]{2}{*}{-1.21} & \multirow[b]{2}{*}{77} & \multirow[b]{2}{*}{.23} & \multirow[b]{2}{*}{-2.38} & \multirow[b]{2}{*}{1.96} \\
\hline & $\begin{array}{l}\text { Plan/Proyecto de intervención, } \\
\text { innovación e investigación }\end{array}$ & 186 & 32.61 & 6.76 & .86 & & & & & \\
\hline \multirow[b]{2}{*}{ Evaluación } & Unidad y programación didáctica & 51 & 25.76 & 5.82 & 1.41 & \multirow[b]{2}{*}{-.71} & \multirow[b]{2}{*}{77} & \multirow[b]{2}{*}{.48} & \multirow[b]{2}{*}{-.88} & \multirow[b]{2}{*}{1.25} \\
\hline & $\begin{array}{l}\text { Plan/Proyecto de intervención, } \\
\text { innovación e investigación }\end{array}$ & 186 & 26.65 & 4.16 & .53 & & & & & \\
\hline \multirow[b]{2}{*}{ Aplicabilidad TFG } & Unidad y programación didáctica & 51 & 22.76 & 6.75 & 1.64 & \multirow[b]{2}{*}{-1.69} & \multirow[b]{2}{*}{77} & \multirow[b]{2}{*}{.09} & \multirow[b]{2}{*}{-3.19} & \multirow[b]{2}{*}{1.88} \\
\hline & $\begin{array}{l}\text { Plan/Proyecto de intervención, } \\
\text { innovación e investigación }\end{array}$ & 186 & 25.95 & 6.92 & .88 & & & & & \\
\hline
\end{tabular}

Tipo TFG = Tipo de TFG; d.t. = Desviación típica; ETM = Error típico de la media; $\mathrm{t}$ = Estadístico $\mathrm{t}$ de Student para muestras independientes; $\mathrm{gl}=$ Grados de libertad; $p=$ Nivel de significación; X1-X2 = Diferencia de medias; DEE = Diferencias del error estándar; (1) Se asumen varianzas iguales.

Fuente: Elaboración propia

\section{Diferencias de valoración del alumnado según la nota media del grado}

Los encuestados que han obtenido en la carrera una nota media de notable o más en la dimensión Proceso de enseñanza-aprendizaje la valoran más $(=11.55$; d.t.=2.93) que los de menos del notable $(=7.60 ;$ d.t. $=4.10)$ existiendo diferencias significativas entre esta dimensión según la nota media de la carrera, $\mathrm{p}=.01<.05$. Por tanto, calculamos el tamaño del efecto (d de Cohen), siendo 1.10, considerándose un tamaño de efecto alto. Además, los encuestados cuya nota media de la carrera sea menos del notable presentan mayor puntuación $(=32.60$; d.t. $=7.50)$ que tuvieron en la carrera de media un notable o superior (=32.07; d.t.=7.20) en tutorización, aunque no existe diferencia significativamente $(\mathrm{p}=.87>.05)$. De la misma manera, los encuestados que obtuvieron en la nota media de la carrera un notable o más, lo valoran más $(=26.58$; d.t. $=4.51)$ que el resto $(=24.60 ;$ d.t. $=2.29)$ en la dimensión Evaluación, aunque sin diferencias estadísticamente significativas $(p=.35>.05)$. En cambio, los encuestados que obtuvieron un notable o más en la carrera $(=25.70$; d.t=6.67) lo valoran mejor que los que obtuvieron menos del notable $(=18.80$; d.t $=8.90$ ) en la dimensión Aplicabilidad TFG, y además existen diferencias significativas con respecto a esta dimensión según la nota media, ya que el p-valor es .03<.05. Por tanto, como existen diferencias significativas entre la dimensión Aplicabilidad TFG con la nota media calculamos el tamaño del efecto ( $d$ de Cohen), siendo .87, considerándose un tamaño de efecto alto (tabla 8). 
Tabla 8 - Diferencias en la valoración del alumnado según la nota media del grado

\begin{tabular}{|c|c|c|c|c|c|c|c|c|c|c|}
\hline Dimensiones & Nota media & $\mathrm{N}$ & Media & d.t. & E.T.M & $\mathrm{T}$ & gl & $\mathrm{p}$ & $\overline{\mathrm{X}} 1-\overline{\mathrm{X}} 2$ & E.T.D \\
\hline \multirow{2}{*}{$\begin{array}{l}\text { Proceso de } \\
\text { E-A del TFG }\end{array}$} & Menos del notable & 15 & 7.60 & 4.10 & 1.83 & \multirow{2}{*}{-2.85} & \multirow{2}{*}{77} & \multirow{2}{*}{.01} & \multirow{2}{*}{-3.95} & \multirow{2}{*}{1.39} \\
\hline & Notable o más & 222 & 11.55 & 2.93 & .34 & & & & & \\
\hline \multirow{2}{*}{ Tutorización } & Menos del notable & 15 & 32.60 & 7.50 & 3.36 & \multirow{2}{*}{.16} & \multirow{2}{*}{77} & \multirow{2}{*}{.87} & \multirow{2}{*}{.53} & \multirow{2}{*}{3.34} \\
\hline & Notable o más & 222 & 32.07 & 7.20 & .84 & & & & & \\
\hline \multirow{2}{*}{ Evaluación } & Menos del notable & 15 & 24.60 & 5.13 & 2.29 & \multirow{2}{*}{-.94} & \multirow{2}{*}{77} & \multirow{2}{*}{.35} & \multirow{2}{*}{-1.98} & \multirow{2}{*}{2.10} \\
\hline & Notable o más & 222 & 26.58 & 4.51 & .52 & & & & & \\
\hline \multirow{2}{*}{ Aplicabilidad TFG } & Menos del notable & 15 & 18.80 & 8.90 & 3.98 & \multirow{2}{*}{-2.20} & \multirow{2}{*}{77} & \multirow{2}{*}{.03} & \multirow{2}{*}{-6.90} & \multirow{2}{*}{3.14} \\
\hline & Notable o más & 222 & 25.70 & 6.67 & .78 & & & & & \\
\hline
\end{tabular}

Nota media en la carrera = Nota media en la carrera; d.t. = Desviación típica; ETM = Error típico de la media; $\mathrm{t}$ = Estadístico $\mathrm{t}$ de Student para muestras independientes; $\mathrm{gl}=$ Grados de libertad; $p=$ Nivel de significación; X1-X2 = Diferencia de medias; DEE = Diferencias del error estándar; (1) Se asumen varianzas iguales.

Fuente: Elaboración propia.

\section{Diferencias de valoración del alumnado según el tipo de centro de realización del grado}

Se observa que existen diferencias significativas en todas las dimensiones según el tipo de centro donde han cursado el TFG. Siendo la mayor diferencia de medias en la dimensión Tutorización, ya que en los centros públicos obtuvieron una puntuación media de 30.88 (d.t.=7.25) mientas que en el privado 35.48 (d.t=5.90). Por tanto, como existen diferencias significativas entre las dimensiones y el tipo de centro calculamos el tamaño del efecto ( $d$ de Cohen), siendo para la dimensión Proceso de enseñanza-aprendizaje del TFG de -.83 (tamaño de efecto débil), para la dimensión Tutorización -.69 (tamaño de efecto débil), para la dimensión Evaluación -.62 (tamaño de efecto débil) -y para la dimensión Aplicabilidad TFG -.63 -tamaño de efecto débil- (tabla 9).

Tabla 9 - Diferencias en el alumnado según el tipo de centro donde está matriculado

\begin{tabular}{|c|c|c|c|c|c|c|c|c|c|c|}
\hline Dimensiones & Centro & $\mathrm{N}$ & Media & d.t. & E.T.M & $t$ & gl & $p$ & $\overline{\mathrm{X}} 1-\overline{\mathrm{X}} 2$ & E.T.D \\
\hline \multirow{2}{*}{$\begin{array}{l}\text { Proceso de } \\
\text { E-A del TFG }\end{array}$} & Público & 197 & 10.67 & 3.10 & .41 & \multirow{2}{*}{-3.14} & \multirow{2}{*}{77} & \multirow{2}{*}{.00} & \multirow{2}{*}{-2.38} & \multirow{2}{*}{.76} \\
\hline & Privado & 40 & 13.05 & 2.58 & .56 & & & & & \\
\hline \multirow{2}{*}{ Tutorización } & Público & 197 & 30.88 & 7.25 & .95 & \multirow{2}{*}{-2.61} & \multirow{2}{*}{77} & \multirow{2}{*}{.01} & \multirow{2}{*}{-4.60} & \multirow{2}{*}{1.76} \\
\hline & Privado & 40 & 35.48 & 5.90 & 1.29 & & & & & \\
\hline \multirow{2}{*}{ Evaluación } & Público & 197 & 25.74 & 4.50 & .59 & \multirow{2}{*}{-2.39} & \multirow{2}{*}{77} & \multirow{2}{*}{.02} & \multirow{2}{*}{-2.69} & \multirow{2}{*}{1.12} \\
\hline & Privado & 40 & 28.43 & 4.14 & .90 & & & & & \\
\hline \multirow{2}{*}{ Aplicabilidad TFG } & Público & 197 & 24.14 & 6.74 & .88 & \multirow{2}{*}{-2.47} & \multirow{2}{*}{77} & \multirow{2}{*}{.02} & \multirow{2}{*}{-4.24} & \multirow{2}{*}{1.72} \\
\hline & Privado & 40 & 28.38 & 6.79 & 1.48 & & & & & \\
\hline
\end{tabular}

Tipo de centro = Tipo de centro; d.t. = Desviación típica; ETM = Error típico de la media; $\mathrm{t}=$ Estadístico $\mathrm{t}$ de Student para muestras independientes; $\mathrm{gl}=\mathrm{Grados}$ de libertad; $p=$ Nivel de significación; X1-X2 = Diferencia de medias; DEE = Diferencias del error estándar; (1) Se asumen varianzas iguales.

Fuente: Elaboración propia. 
En cuanto a las correlaciones de Pearson (tabla 10) podemos observar que la dimensión Proceso de enseñanza-aprendizaje del TFG tiene correlaciones significativas con el resto de las dimensiones, existiendo la relación mayor con la dimensión Aplicabilidad TFG (.642), siendo una relación directa y positiva. Además, la dimensión Tutorización está correlacionada significativamente con el resto de dimensiones, siendo la mayor correlación significativa, directa y positiva con la dimensión Proceso de enseñanza-aprendizaje del TFG. Y la dimensión Evaluación está correlacionada significativamente con el resto de dimensiones, al igual que ocurre con la dimensión Aplicabilidad TFG. Podemos concluir que todas las dimensiones están correlacionadas entre sí, siendo todas las correlaciones directas y positivas.

Tabla 10 - Correlaciones Pearson entre las dimensiones de nuestro estudio

\begin{tabular}{|c|c|c|c|c|}
\hline Correlaciones Pearson & Proceso de E-A del TFG & Tutorización & Evaluación & Aplicabilidad TFG \\
\hline Proceso de E-A del TFG & 1 & $.614^{* *}$ & $.559^{* *}$ & $.642^{* *}$ \\
\hline Tutorización & $.614^{* *}$ & 1 & $.514^{* *}$ & $.380^{* *}$ \\
\hline Evaluación & $.559^{* *}$ & $.514^{*+}$ & 1 & $.516^{* *}$ \\
\hline Aplicabilidad TFG & $.642^{* *}$ & $.380^{*+}$ & $.516^{*+}$ & 1 \\
\hline
\end{tabular}

** La correlación es significativa en el nivel 0.01 (2 colas).

Fuente: Elaboración propia.

Por otro lado, de las correlaciones de Spearman (tabla 11), se puede corroborar que la dimensión Proceso de enseñanza-aprendizaje del TFG tiene correlaciones significativas con el resto de las dimensiones, existiendo la relación directa y positiva con la dimensión Tutorización (.644). Así mismo, la dimensión Tutorización también correlaciona significativamente con el resto de dimensiones, siendo la correlación más significativa con la dimensión Proceso de enseñanza-aprendizaje del TFG. Por último, las dimensiones Evaluación y Aplicabilidad están correlacionadas significativamente con el resto de dimensiones. Por lo tanto, se puede afirmar que todas las dimensiones están correlacionadas entre sí.

Tabla 11 - Correlaciones de Spearman entre las dimensiones de nuestro estudio

\begin{tabular}{|c|c|c|c|c|}
\hline Correlaciones de Spearman & Proceso de E-A del TFG & Tutorización & Evaluación & Aplicabilidad TFG \\
\hline Proceso de E-A del TFG & 1 & $.644^{\star *}$ & $.543^{\star *}$ & $.630^{\star *}$ \\
\hline Tutorización & $.644^{\star *}$ & 1 & $.512^{\star \star}$ & $.388^{\star *}$ \\
\hline Evaluación & $.543^{\star *}$ & $.512^{\star *}$ & 1 & $.497^{\star \star}$ \\
\hline Aplicabilidad TFG & $.630^{\star \star}$ & $.388^{\star *}$ & $.497^{\star \star}$ & 1 \\
\hline
\end{tabular}

\footnotetext{
** La correlación es significativa en el nivel 0.01 ( 2 colas).
}

Fuente: Elaboración propia. 
A continuación, en el análisis de regresión, cabe destacar que en las dimensiones Evaluación y Proceso de enseñanza-aprendizaje del TFG existe un valor atípico, que está por debajo del valor mínimo de ambas dimensiones (gráfico 6). En nuestro estudio, los valores atípicos son muy cercanos a los valores mínimos que toman las dimensiones, por lo que no merece la pena eliminar el valor de los datos (ya que las dimensiones han sido calculadas como suma de varios factores). El resto de dimensiones (Tutorización y Aplicabilidad del TFG) no tienen ningún valor atípico, por lo que no existe ningún error de entrada de datos o de medición.

Gráfico 5 - Gráfico de regresión

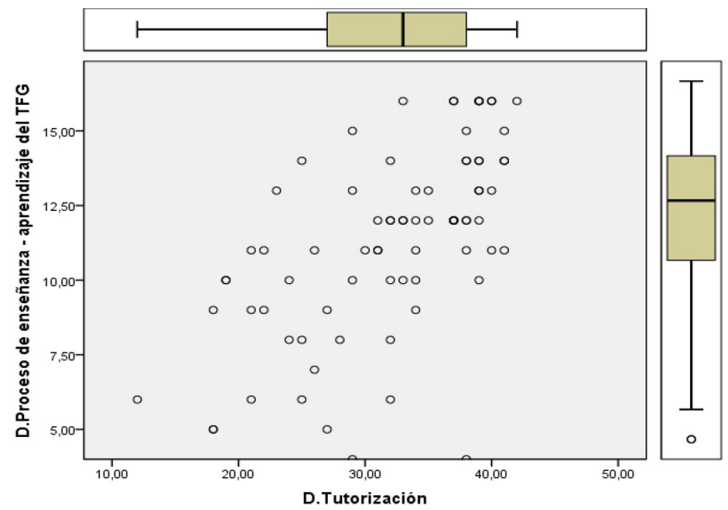

Fuente: Elaboración propia.

Gráfico 7 - Gráfico de regresión

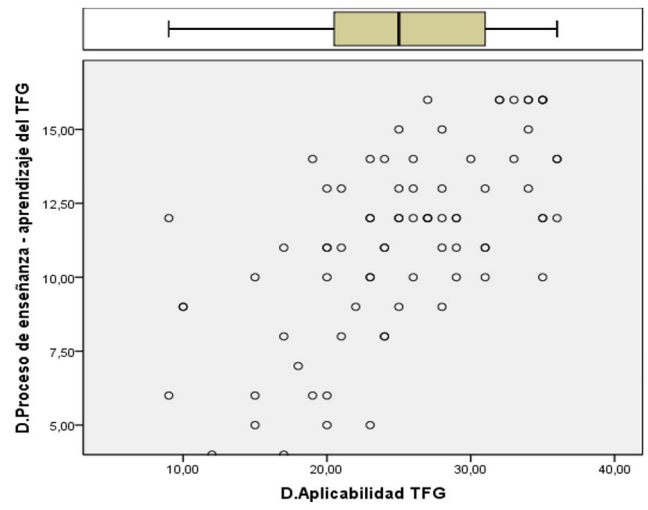

Fuente: Elaboración propia.
Gráfico 6 - Gráfico de regresión

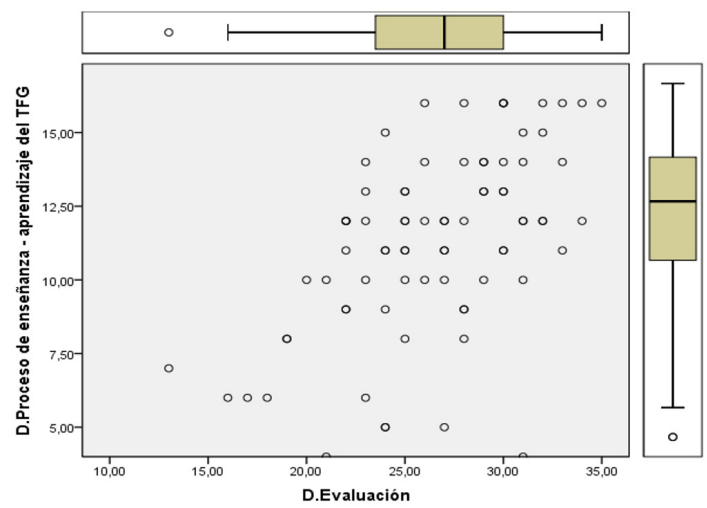

Fuente: Elaboración propia.

Gráfico 8 - Gráfico de regresión

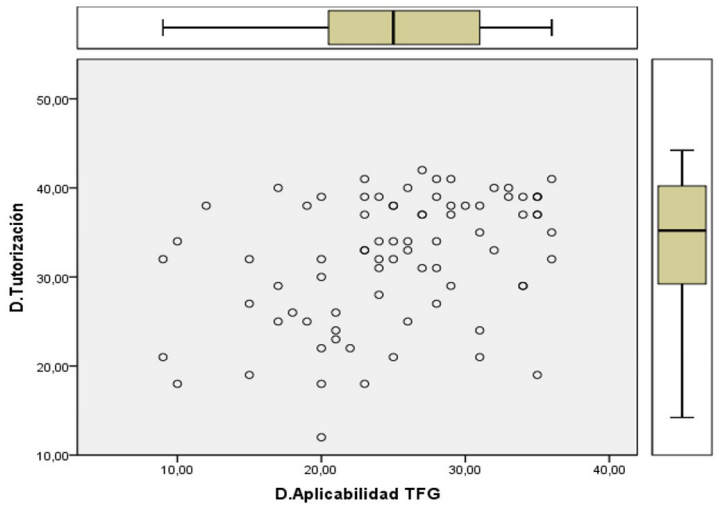

Fuente: Elaboración propia. 
Gráfico 9 - Gráfico de regresión

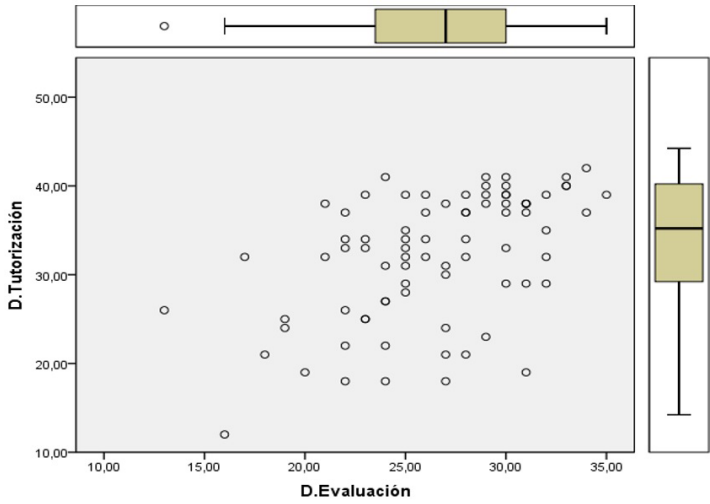

Fuente: Elaboración propia.

Gráfico 11 - Gráfico de regresión

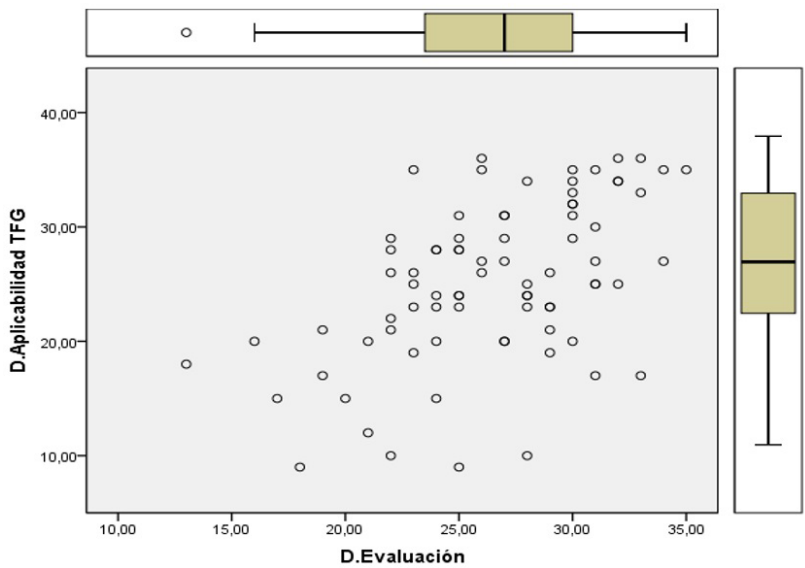

Fuente: Elaboración propia.
Gráfico 10 - Gráfico de regresión

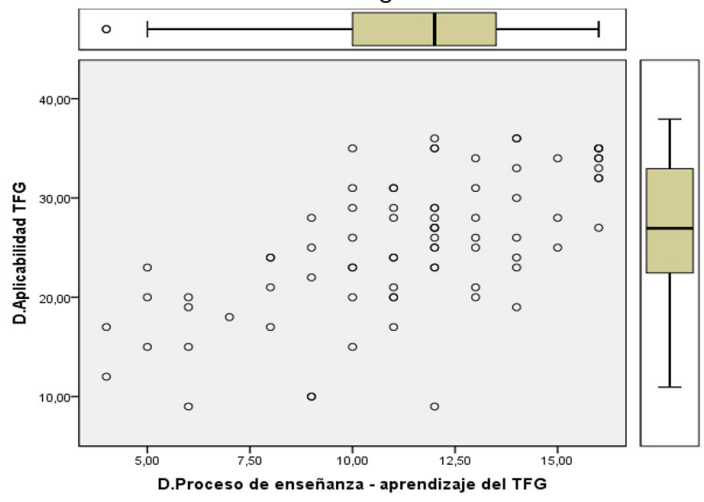

Fuente: Elaboración propia.

\section{Discusión y conclusiones}

De los resultados obtenidos, destacamos ciertas tendencias a modo de conclusión. Así, se puede afirmar que existe una correlación directa y positiva entre todos los aspectos preguntados y las diferentes dimensiones, es decir, el cuestionario ofrece coherencia en relación con los diversos ámbitos analizados para dicha asignatura.

La relevancia de nuestro trabajo ha sido confirmar por la voz que tiene nuestro alumnado de los procesos que traen implícitos la realización del Trabajo Fin de Grado, tanto para el alumnado como para el profesorado; ya que, como sugieren Susinos y Calvo (2010), la investigación que escucha la voz del alumnado repercute en el proceso de mejora de la institución. 
Teniendo presentes los objetivos de la investigación, se ha comprobado que no existen diferencias según el género de los estudiantes en las respuestas dadas a las distintas dimensiones. Por el contrario, sí se aprecian diferencias según la edad, en cuanto a la dimensión Aplicabilidad del TFG, debido a la mayor motivación intrínseca de los participantes mayores de 30 años y al uso de estrategias de estudio más profundas -basadas en la comprensión y en la realización de trabajos- (MAS; MEDINAS, 2007).

Igualmente no existen diferencias en las valoraciones sobre el TFG en el alumnado según la nota de acceso al grado, aunque los que tienen menos nota valoran más positivamente la dimensión de la Tutoría. En consecuencia, la acción tutorial basada en tutorías programadas para fijar las líneas argumentales (DOPICO, 2013), sigue siendo la más demandada por el alumnado. Así mismo, tampoco existen diferencias significativas entre los estudiantes según el tipo de TFG realizado y la labor tutorial del profesorado.

En esta misma línea, según la nota media obtenida por los estudiantes en sus titulaciones, existen diferencias significativas con la dimensión Aplicabilidad, a favor del alumnado que tiene en el grado una nota media de notable o superior, explicación que estaría relacionada con las investigaciones comentadas anteriormente sobre la motivación intrínseca.

Por otro lado, vemos como según el tipo de centro donde están matriculados, existen diferencias significativas a favor del centro privado en todas las dimensiones, destacando la mayor diferencia en la dimensión de la Tutoría (CAÑAS; CAMPOY; PANTOJA, 2005). Profundizando en esta afirmación vemos como la Tutoría en ambos centros alcanza una leve valoración positiva, mayor que el resto de las dimensiones en las medias obtenidas, coincidiendo con otros estudios realizados (VERA; BRIONES, 2014; VERA; BRIONES; SOTOS, 2014) y si la Tutoría incluye aspectos tan importantes como las aportaciones que realizan los tutores para conocer la estructura, la presentación, el marco teórico o el planteamiento metodológico, nos parecen relativamente positivas. Por ello, pensamos que el proceso de tutorización que se realiza necesita una revisión, ya que el alumnado, aun valorando la labor tutorial positivamente, no muestra una aceptación rotunda de dicha labor, en consecuencia, el tutor debe profundizar más en desarrollar las competencias profesionales que les vienen prescritas para ejercer esta labor (PINED0, 2016), sabiendo que es un proceso en el que también intervienen elementos personales, psicológicos e interrelacionales (KOSTER; DENGERINK, 2008). Las tutorías deben ser programadas como labor inherente a la función del profesorado y con una implicación mayor por parte del mismo. Es por ello que se considera necesario una reformulación de las funciones del tutor del TFG (MUÑOZ, 2015).

En relación con la dimensión Aplicabilidad, el alumnado valora relativamente la utilidad y el sentido práctico del TFG, pero es una asignatura que conlleva una implicación muy superior a las demás. Hay que destacar que el alumnado ve este trabajo con cierta complejidad; aunque el Practicum les ayuda en su realización (ALVAREZ; ORDOÑEZ, 2017; CORCELLES et al., 2017). Así mismo, destacamos que no valoran la asignatura como la más enriquecedora, aunque han ampliado sus conocimientos académicos y han percibido una mejora profesional y personal durante su desarrollo.

En esta misma línea, la dimensión Aplicabilidad correlaciona con la dimensión de Evaluación, y debe ser entendida como la explicación lógica a la necesidad de proponer 
un modelo coherente de Evaluación con el que el alumnado se sienta identificado (VILLAMAÑE et al., 2017). En relación con la dimensión de Evaluación, aunque la valoran de forma positiva, también consideran que el sistema de evaluación no es del todo adecuado y no muy justo (aspecto que se ha modificado en la nueva normativa de la Universidad de Jaén: actualmente la evaluación es realizada únicamente por el tutor). Además, el alumnado ve necesario valorar también otras competencias transversales (uso de las TIC, aprender a aprender, etc.). El TFG es la antesala de su futuro profesional donde la práctica debe avanzar con la reflexión-acción y por ello, hay que dotar al alumnado de estrategias para realizarlas, investigarla y/o saber modificarla (INAREJOS, 2017) en relación de los resultados. Es por eso que se necesita en los Grados de Educación más asignaturas y contenidos relacionados con la investigación educativa. Así mismo, debido a la necesidad de incrementar la utilidad de lo trabajado, el TFG debe ser enfocado hacia la profesionalidad y con un marcado carácter interdisciplinar a lo largo del Grado (RUBIO et al., 2018). La educación necesita constatar buenas prácticas de éxito y fomentarlas. El profesional de la educación no puede hacer lo que le dicen que haga, sino que debe experimentar lo que verdaderamente es exitoso para su aula.

En cuanto a la valoración de la dimensión Proceso de enseñanza-aprendizaje no es muy valorada ni considerada por los mayores de 30 años, ya que consideran que, aunque el TFG ha aumentado sus conocimientos, les ha superado en relación con otros trabajos desarrollados en el grado. Se debe pensar que, como hemos visto, no han desarrollado lo suficiente las competencias relacionadas con dicho proceso. Por otro lado, el alumnado percibe que el trabajo no lo ha co-realizado con su tutor y que no se han cumplido sus expectativas académicas, sea cual sea el tipo de trabajo realizado. Las competencias propias de TFG destacan la necesidad de que el alumnado sea capaz de aprender a aprender, no obstante, si durante el grado estas competencias no se desarrollan o al menos se inician, es lógico pensar en la dificultad a la que se enfrenta el alumnado. El esfuerzo de los docentes debe ir encaminado a repensar e innovar las dinámicas en el aula y programar actividades de aprendizaje prácticas que ayuden en el proceso de desarrollo competencial (TORRAD0; REGUANT, 2016), que favorezcan el compromiso e implicación del estudiante en su propio proceso formativo de aprender a aprender.

Con los avances de este estudio no queremos extrapolar los resultados, ya que somos conscientes de las limitaciones propias de un contexto limitado y con una muestra factible de ser ampliada. Por ello, en futuros estudios, creemos conveniente seguir recogiendo información sobre la voz de nuestro alumnado en otros momentos académicos y en otros contextos (SUSINOS; CEBALLOS, 2012), como proceso de mejora y cambio en la Educación Superior.

\section{Referencias}

ALVAREZ, Juan Carlos; ORDOÑEZ, Iluminada. Prácticum y prácticas docentes: la intervención de los profesionales en la docencia. Lan Harremanak, Leioa, v. 37, p. 83-94, 2017.

AMADOR, Maria Isabel; SERRANO, Rocío. Guía práctica para el trabajo de fin de grado en educación. Barcelona: Grao, 2017. 
BARTOLOMÉ, Antonio et al. El trabajo de fin de grado: guía para estudiantes, docentes y agentes colaboradores. Madrid: McGraw Hill, 2012.

BONILLA, María Isabel; MARTíN, Carolina. Evaluación de competencias en el trabajo fin de grado en administración y dirección de empresas: una propuesta de la Facultad de Ciencias Sociales de Talavera de la Reina. UCLM. Revista de Formación e Innovación Educativa Universitaria, Vigo, v. 5, n. 4, p. 241-253, 2012.

CAÑAS, Antonio; CAMPOY, Tomás; PANTOJA, Antonio. La función tutorial: valoración y necesidades del profesorado. Bordón, Madrid, v. 57, n. 3, p. 297-314, 2005.

COHEN, Jacob. Statistical power analysis for the behavioral sciences. New Jersey: Lawrence Erlbaum, 1998.

CORCELLES, Mariona et al. Enseñar a escribir un artículo de investigación mediante la revisión colaborativa: percepciones de los estudiantes. Revista Signos, Viña del Mar, v. 50, n. 95, p. 337-360, 2017.

DE PRO, Antonio.; SÁNCHEZ, Gaspar; VALCÁRCEL, Ma Victoria ¿En qué medida están contribuyendo los TFM a los resultados de aprendizaje planificados? Revista Eureka sobre Enseñanza y Divulgación de las Ciencias, Cádiz, v. 10, p. 728-748, 2013.

DE VAUS, David. Surveys in social research. London: Routledge: Gideon, 2002.

DíEZ, Emiliano et al. Espacio europeo de educación superior: estándares e indicadores de buenas prácticas para la atención a estudiantes universitarios con discapacidad. Salamanca: Inico, 2011.

DOPICO, Eduardo. Tutoría universitaria: propuestas didácticas de competencia tutorial. Redu, Valencia, v. 11, n. 2, p. 195-220, 2013.

ESPAÑA. Orden ECl/3857, de 27 de diciembre de 2007. Requisitos para la verificación de los títulos universitarios oficiales que habiliten para el ejercicio de la profesión de maestro en educación primaria. Boletín Oficial del Estado de España, Madrid, n. 312, p. 53747-53750, 29 dici. 2007.

ESPAÑA. Real Decreto 43, de 2 de febrero de 2015. Modificación del Real Decreto 1393/2007 por el que se establece la ordenación de las enseñanzas universitarias oficiales, y del Real Decreto 99/2011 por el que se regulan las enseñanzas oficiales de doctorado. Boletín Oficial del Estado de España, Madrid, n. 29, p. 8088-8091, 03 febr. 2015.

ESPAÑA. Real Decreto 1393, de 29 de octubre de 2007. Ordenación de las enseñanzas universitarias oficiales. Boletín Oficial del Estado de España, Madrid, n. 260, p. 44037-44048, 30 oct. 2007.

FERRER, Virginia; CARMONA, Moisés; SORIA, Vanessa. El trabajo de fin de grado: guía para estudiantes, docentes y agentes colaboradores. Madrid: McGraw Hill, 2012.

FONDEVILA, Joan Francesc; OLMO, José Luis. El trabajo de fin de grado en ciencias sociales y jurídicas: guía metodológica. Madrid: Internacionales Universitarios, 2013. 
GARCÍA, Ma Paz; MARTÍNEZ, Pilar. Guía práctica para la realización de trabajos de fin de grado y trabajos fin de máster. Murcia: Editum, 2012.

GONZÁLEZ, Juan Mª LEÓN, Ana; PEÑALBA, Mercedes. Cómo escribir un trabajo de fin de grado. Madrid: Síntesis, 2014.

GONZÁLEZ, Nuria; ABAD, Julio Ignacio; LEVY, Jean Pierre. Modelización con estructuras de covarianzas. La Coruña: Netbiblo, 2006.

GUTIÉRREZ, Miguel Ángel; LÓPEZ, Alfonso. Gestión documental en trabajos fin de grado: uso de gestores documentales y flujos de trabajo. Madrid: EAE, 2012.

INAREJOS, Juan Antonio. El uso de fuentes históricas y bases documentales en la formación del profesorado de educación infantil y primaria. Revista Electrónica Interuniversitaria de Formación del Profesorado, Murcia, v. 20, n. 2, p. 157-166, 2017.

KOSTER, Bob; DENGERINK, Jurriën. Professional standards for teacher educators: how to deal with complexity, ownership and function: experiences from the Netherlands. European Journal of Teacher Education, London, v. 31, n. 2, p. 135-149, 2008.

LATORRE, Antonio; DEL RINCÓN, Delio; ARNAL, Justo. Bases metodológicas de la investigación educativa. Barcelona: Experiencia, 2003.

LATORRE, Ma Pilar. Guía del trabajo de fin de grado adaptado al EEES. Zaragoza: Stylo Digital, 2011.

LAVRAKES, Paul. Encyclopedia of survey research methods. Thousand Oaks: Sage, 2008.

MARTíNEZ, Miguel Ángel et al. Estadística amigable. Barcelona: Elsevier, 2014.

MAS, Carmen; MEDINAS, Magdalena. Motivaciones para el estudio en universitarios. Anales de Psicología, Murcia, v. 23, n. 1, p. 17-24, 2007.

MATEO, Joan. Guía para la evaluación de competencias en el trabajo de fin de grado en el ámbito de las ciencias sociales y jurídicas. Barcelona: AQU Catalunya, 2009.

MORENO, Verónica; HERNÁNDEZ, Davinia. Rubric-based tools to support the monitoring and assessment of bachelor's final projects/herramientas basadas en rúbricas para el control y la evaluación de los proyectos final de grado. Education in the Knowledge Society, Salamanca, v. 16, n. 4, p. 47, 2015.

MUÑIZ, José; FONSECA, Eduardo. Construcción de instrumentos de medida para la evaluación universitaria. Revista de Investigación en Educación, Vigo, n. 5, p. 13-25, 2009.

MUÑOZ, Gemma. Cómo elaborar y defender un trabajo académico en humanidades: del trabajo de fin de grado al trabajo de fin de máster. Madrid: Bubok, 2015. 
PEPPER, David; WEBSTER, Frank; JENKINS, Alan. Benchmaking in geography: some implications for assessing dissertations in the undergraduate curriculum. Journal of Geography in Higher Education, London, v. 25, n. 1, p. 23-35, 2001.

PÉREZ, José Manuel; MARTíNEZ, Sergio. Ideas alternativas sobre el trabajo fin de grado en estudiantes de magisterio. ReiDoCrea, Granada, n. 6, p. 246-259, 2017.

PINED0, Ruth. Competencias profesionales del tutor del trabajo fin de grado. Revista INFAD de Psicología "International Journal of Developmental and Educational Psychology", Extremadura, v. 4, n. 1, p. 329-334, 2016.

REYES, Carmen Isabel; DÍAZ, Alicia. ¿Se adecúa la normativa de evaluación del trabajo fin de grado a las exigencias del espacio Europeo de educación superior? Revista Complutense de Educación, Madrid, v. 28, n. 4, p.1285, 2017.

RODRÍGUEZ, Itziar. ¿Cómo afrontar el trabajo fin de grado? un problema o una oportunidad para culminar con el desarrollo de las competencias. Revista Complutense de Educación, Madrid, v. 22, n. 2, p. 179-193, 2012.

RODRÍGUEZ, Rocío et al. La tutoría piramidal como estrategia de aprendizaje para el diseño y desarrollo del trabajo final de grado en educación. Revista de Innovación y Buenas Prácticas Docentes, Córboda, n. 3, p. 68-75, 2017.

RUBI0, María José et al. Autopercepción de las competencias investigativas en estudiantes de último curso de pedagogía de la Universidad de Barcelona para desarrollar su trabajo de fin de grado. Revista Complutense de Educación, Madrid, v. 29, n. 2, p. 335-354, 2018.

RULLÁN, Mercedes et al. La evaluación de competencias transversales en la materia trabajos fin de grado: un estudio preliminar sobre la necesidad y oportunidad de establecer medios e instrumentos por ramas de conocimiento, REDU, Valencia, v. 8, n. 1, p. 74, 2010.

SÁNCHEZ, Fermín et al. El método socrático como guía del trabajo de fin de grado. Revista de Investigación en Docencia Universitaria de la Informática, Castellón, v. 8, n. 1, p. 53-62, 2015.

SOTOS, Ma Antonia. Trabajo fin de grado: ¿Oportunidad o trámite? Jornadas sobre el Aprendizaje y la Enseñanza de las Matemáticas, Cartagena, 2015. Disponible en: <https://ruidera.uclm.es/xmlui/ handle/10578/8956>. Acceso en: 10 en. 2018.

SUSINOS, Teresa; CALVO, Adelina. Prácticas de investigación que escuchan la voz del alumnado: mejorar la universidad indagando la experiencia. Profesorado, Revista de Currículum y Formación del Profesorado, Granada, v. 14, n. 3, p. 75-88, 2010.

SUSINOS, Teresa; CEBALLOS, Noelia. Voz del alumnado y presencia participativa en la vida escolar: apuntes para una cartografía de la voz del alumnado en la mejora educativa. Revista de Educación, n. 359, p. 24-44, 2012. Disponible en: <http://www.mecd.gob.es/dctm/revista-de-educacion/articulosre359/re35902.pdf? documentld=0901e72b813d7295>. Acceso en: 3 nov. 2015. 
TORRAD0, Mercedes; REGUANT, Mercedes. Las actividades de aprendizaje y su contribución en el desarrollo competencial en investigación educativa: el caso del grado de pedagogía de la UB. Educatio Siglo XXI, Murcia, v. 34, n. 2, p. 9-32, 2016. D0I: http://dx.doi.org/10.6018/j/263791

TUR, Victoria et al. Planificación y evaluación de la asignatura trabajo fin de máster. Alicante: ICE, 2013.

VALDERRAMA, Elena et al. La evaluación de competencias en los trabajos fin de estudios. IEEE-RITA, Vigo, v. 5, n. 3, p. 107-114, 2010.

VERA, Jesús; BRIONES, Elena. Elementos clave del proceso de tutorización y evaluación del TFG: análisis y resultados en la UVA y la UC. San Sebastián: Universidad del País Vasco, 2014.

VERA, Jesús; BRIONES, Elena. Perspectiva del alumnado de los procesos de tutorización y evaluación de los trabajos de fin de grado. Cultura y Educación, Madrid, v. 27, n. 4, p. 726-765, 2015.

VERA, Jesús; BRIONES, Elena; SOTOS, María. Los procesos de tutorización y evaluación del trabajo de fin de grado (TFG): un estudio de dos años de seguimiento (2011/12-2012/13). Bilbao: Universidad del País Vasco, 2014. Disponible en: <http://goo. gl/sfl1B9>. Acceso en: 12 febr. 2016.

VILLA, Aurelio; POBLETE, Manuel. Aprendizaje basado en competencias: una propuesta para la evaluación de las competencias genéricas. Bilbao: Universidad de Deusto, 2007.

VILLAMAÑE, Mikel et al. Desarrollo y validación de un conjunto de rúbricas para la evaluación de trabajos fin de grado. ReVisión, Castellón, v. 10, n. 1, 2017.

ZABALZA Miguel Ángel. Competencias docentes del profesorado universitario: calidad y desarrollo profesional. Madrid: Narcea, 2006.

ZAIRI, Mohamed. Managing customer satisfaction: a best practice perspective. The TQM Magazine, West Yorkshire, v. 12, n. 6, p. 389-394, 2000.

Recibido en: 23.05 .2018 Modificaciones en: 21.08.2018

Aprobado en: 27.11.2018

Javier Rodríguez Moreno es doctor en Educación por la Universidad de Murcia (España). Profesor del Departamento de Pedagogía de la Universidad de Jaén (España).

María Dolores Molina Jaén es doctora en Pedagogía por la Universidad de Jaén (España). Profesora del Departamento de Psicopedagogía del Centro Universitario Sagrada Familia -adscrita a la Universidad de Jaén- (España).

María Jesús Colmenero Ruiz es doctora en Filosofía y Ciencias de la Educación por la Universidad de Jaén (España). Profesora del Departamento de Pedagogía de la Universidad de Jaén (España). 